\title{
Surface-bounded growth modeling applied to human mandibles
}

Andresen, Per Rønsholt; Brookstein, F. L.; Conradsen, Knut; Ersbøll, Bjarne Kjær; Marsh, J.; Kreiborg, S.

Published in:

I E E E Transactions on Medical Imaging

Link to article, DOI:

$10.1109 / 42.896780$

Publication date:

2000

Document Version

Publisher's PDF, also known as Version of record

Link back to DTU Orbit

Citation $(A P A)$ :

Andresen, P. R., Brookstein, F. L., Conradsen, K., Ersbøll, B. K., Marsh, J., \& Kreiborg, S. (2000). Surfacebounded growth modeling applied to human mandibles. I E E E Transactions on Medical Imaging, 19(11), 10531063. https://doi.org/10.1109/42.896780

\section{General rights}

Copyright and moral rights for the publications made accessible in the public portal are retained by the authors and/or other copyright owners and it is a condition of accessing publications that users recognise and abide by the legal requirements associated with these rights.

- Users may download and print one copy of any publication from the public portal for the purpose of private study or research.

- You may not further distribute the material or use it for any profit-making activity or commercial gain

- You may freely distribute the URL identifying the publication in the public portal 


\title{
Surface-Bounded Growth Modeling Applied to Human Mandibles
}

\author{
Per Rønsholt Andresen*, Fred L. Bookstein, Knut Conradsen, Bjarne Kjær Ersbøll, Jeffrey L. Marsh, and \\ Sven Kreiborg
}

\begin{abstract}
From a set of longitudinal three-dimensional scans of the same anatomical structure, we have accurately modeled the temporal shape and size changes using a linear shape model. On a total of 31 computed tomography scans of the mandible from six patients, 14851 semilandmarks are found automatically using shape features and a new algorithm called geometry-constrained diffusion. The semilandmarks are mapped into Procrustes space. Principal component analysis extracts a one-dimensional subspace, which is used to construct a linear growth model. The worst case mean modeling error in a cross validation study is $3.7 \mathrm{~mm}$.
\end{abstract}

Index Terms-Geometry-constrained diffusion, morphometrics, nonrigid shape-preserving registration, principal component analysis, Procrustes analysis, semilandmarks.

\section{INTRODUCTION}

$\mathbf{P}$ EDIATRIC craniofacial surgeons need insight into expected facial growth. This paper is concerned with the mandible, a particularly complex bony structure both in its shape and in its growth process, as two sets of teeth erupt asynchronously while the direction of the condylar process changes by a considerable angle.

Our data set here comprises 31 mandibular surfaces extracted from computed tomography (CT) scans of a total of six children diagnosed with Apert syndrome.

The analysis falls under two major headings. First is the representation of the set of mandibular surfaces by one vector of 14851 points that can be considered to be semilandmarks: that is, points that do not have names, but that correspond across all the cases of a data set under a reasonable model of deformation from their common mean [1]. Second is the summary of these

Manuscript received August 3, 1999; revised August 17, 2000. This paper was supported by the John and Birthe Meyer Foundation. The work of P. R. Andresen was supported by the Danish Technical Research Council under Grant 9600452 . The work of F. Bookstein was supported by the United States Public Health Service (USPHS) under Grant GM-37 251 to the University of Michigan. The Associate Editor responsible for coordinatinating the review of this paper and recommending its publication was M. W. Vannier. Asterisk indicates corresponding author.

*P. R. Andresen is with the Section for Image Analysis at Department of Mathematical Modeling, Technical University of Denmark, DK-2800 Lyngby, Denmark (e-mail: pra@imm.dtu.dk).

F. L. Bookstein is with the Institute of Gerontology, University of Michigan, Ann Arbor, MI, 48109 USA.

K. Conradsen and B. K. Ersbøll are with the Section for Image Analysis at Department of Mathematical Modeling, Technical University of Denmark, DK-2800 Lyngby, Denmark.

J. L. Marsh is with the Division of Plastic and Reconstructive Surgery, Department of Surgery, Washington University School of Medicine, St. Louis, MO 63130 USA.

S. Kreiborg is with the Department of Pediatric Densistry, University of Copenhagen, DK-2200 Copenhagen, Denmark.

Publisher Item Identifier S 0278-0062(00)09748-2.
31 point sets in a linear subspace of surprisingly low dimension, affording surprisingly accurate growth predictions.

The organization of the remainder of this text is as follows. Section II sets the stage for our algorithm by reviewing the literature of nonrigid registration by deformable models. Section III introduces the patients to which we have access, their CT images, and the mechanism by which we produced the 31 mandibular surfaces of the data set. Section IV describes the initialization of our diffeomorphism by detection and matching of crest lines, and Section V shows how we proceed to a full surface-constrained diffusion. In Sections VI and VII, the (31 1)-dimensional sample subspace of the full space of semilandmark shape is subjected to certain conventional multivariate biometric analyses that yield powerful predictors of unobserved (future) form. We assess their accuracy using measures of surface-to-surface discrepancy. Section VIII is a retrospect over all these tactics, emphasizing the surprising power of the diffusion methodology to uncover commonalities in the six independent growth processes of this sample. We close with a plea for corresponding studies of normative samples.

\section{RELATED WORK}

The literature treating registration methods is very extensive (e.g., [2] or [3] for surveys). This section, therefore, mainly concentrates on the literature covering both registration and deformable models (for reviews, e.g., [4] or [5]) or morphometrics (e.g., [6] and [7]). We will emphasize the registration method of Feldmar and Ayache [8], as it most resembles the geometry-constrained diffusion method [9], [10]. Feldmar and Ayache [8] perform a surface registration based on a distance measure that relies on local geometrical properties of the surfaces. The surface registration is a collection of local affine registrations, spatially blurred so as to result in a smoothly varying global registration. Geometry-constrained diffusion (Section V) does not make a collection of local affine frames, but a global registration field. We do not exploit any metric properties of the surfaces, but look for a globally simple registration field. This also creates a tendency to match points of similar geometry since the field, otherwise, must be more complex.

Deformable models have been widely studied [4], [5]. When using landmarks to drive the correspondence between objects one main drawback is the the need for their manual location in advance. A lot of researchers have worked on this. Bookstein has reported a method where the semilandmarks are placed on contours automatically [1]. Note, a landmark is a point that can be identified by verbal characterization on the single case [11]. 


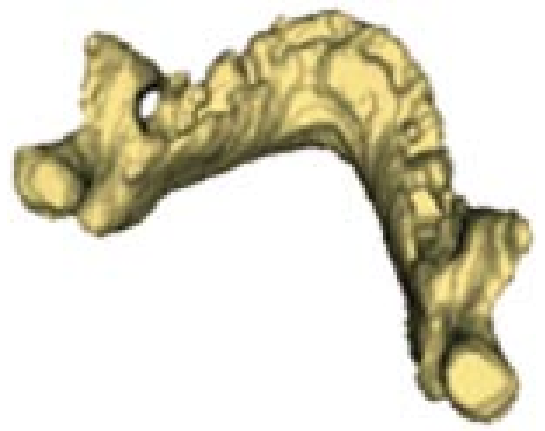

Fig. 1. The raw dataset of patient 3, scan \#1 (1-mo scanning). The mandibular bone and teeth have low density, therefore, cavities and holes are introduced when segmented.
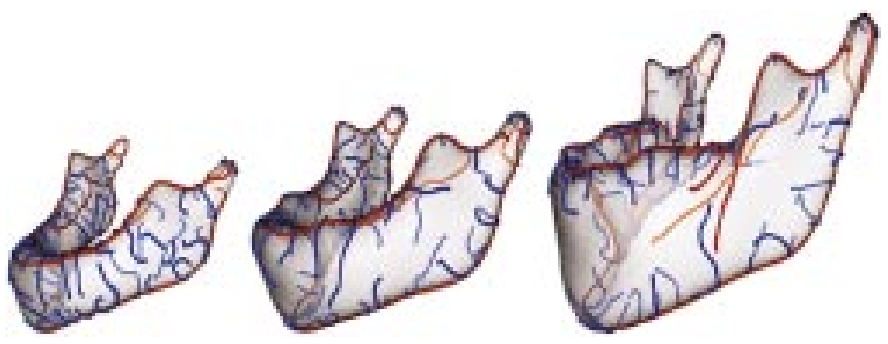

Fig. 2. The crest lines (in red) and $k_{2}$-max lines (in blue) on the three Gaussian smoothed (kernel size, $\sigma=3 \mathrm{~mm}$ ) mandibles at (left) 9 mo, (middle) $21 \mathrm{mo}$, and (right) 7 -yr old. The mandibles are from patient 6, scan \#1, \#2, and \#3, respectively. The surfaces are translucent.

Semilandmarks are points that do not have names, but correspond across all the cases as images of the same point of their average, so one can carry out statistics just as if they were landmark points [1].

Fleute and Lavallée [12] extrapolate a small number of range data to obtain a complete surface representation. Principal component analysis (PCA) is used to reduce the dimensionality. Data sets are registered together using an elastic registration method of Szeliski and Lavallée [13] based on octree-splines. The method is a least squares minimization of the distances between a sparse and unorganized set of points and a dense set used to build a three-dimensional (3-D) octree-spline distance map. The result is a smooth deformation field.

A registration technique based on thin-plate splines that takes landmark errors into account has been reported in [14]. The semilandmarks are located semiautomatically or manually.

The present work has been greatly inspired by the seminal work of Cootes and colleagues ([15] for an overview) and Dean et al. [16] (see below). Manually detected landmarks have been used for Cootes and colleagues' analysis [15]. Principal components are calculated from the Procrustes analysis and an active shape model is made. The deformation of the active shape model is restricted by the principal components. For segmentation, gray-level information near the object boundaries is also modeled.

Kelemen et al. [17] have used the same method as in [15], but in order to automate the landmark generation, Fourier-descriptors [18], [19] were found very powerful. Restrictions on the topology of the surfaces are the main drawback when using Fourier descriptors.

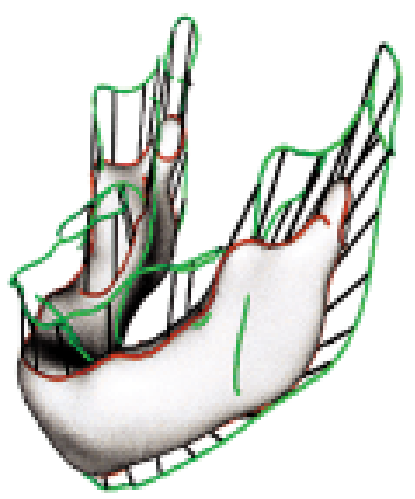

Fig. 3. Matches (lines in black) between two sets of crest lines at scale 3 . The crest lines on the 21-mo and 7-yr-old mandible (patient 6, scan \#2 and $\# 3$, respectively) are red and green, respectively. For visual clarity, only every eighth match is shown.

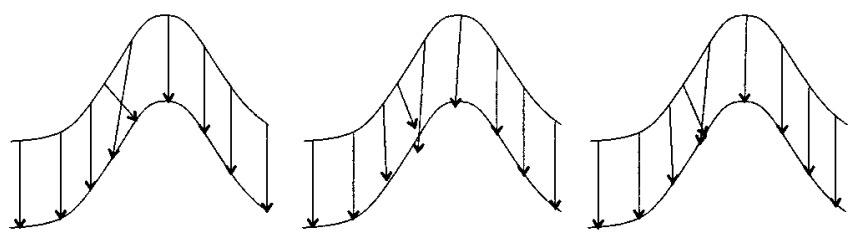

Fig. 4. The images show schematically how the diffusion algorithm works on the displacement field. The Cartesian components of the initial displacement field (arrows in the left image) are Gaussian smoothed. Some of the links have now diverged from the surface (middle image) and must be projected back on to the surface (right image). The fold is removed by repeating the steps until the field does not change.

In the present paper, the registration method of Subsol et al. [20] gives the initial object correspondence (Section IV). Crest lines [21] are registered together taking into account the constraints inferred by lines and a heuristic algorithm based on the iterative closest-point (ICP) algorithm [22].

Grenander and Miller [23] have formalized a model of anatomy in which anatomies are represented as deformable templates, collections of zero-, one-, two-, and three-dimensional manifolds. They have three principal themes in computational anatomy: large deformations maps [24], emperical probability laws that represent anatomical variation in populations, and inferences on populations, such as disease classification.

Lorenz and Krahnstöver [25] present a 3-D shape model based on surface points. The overall strategy is similar to that in the present paper. However, the initial correspondence is based on a manually defined set of landmarks on each object. From the sparse mapping defined by the landmarks, a dense mapping is calculated by thin-plate interpolation. If it happens that the deformed mesh is folded, it is unfolded using a mesh relaxation balancing bending force aginst surface mesh force by a mass-spring model.

Dean et al. [16] analyze pairs of frontal and lateral X-rays from 32 individuals (16 males and 16 females) aged from 3to 18 -yr old. The objective is to investigate which 3-D landmarks could be collected with high precision, to identify ontogenetic trends in landmark configuration shape change, and to detect patterns of sexual dimorphism. The 32 landmarks are 


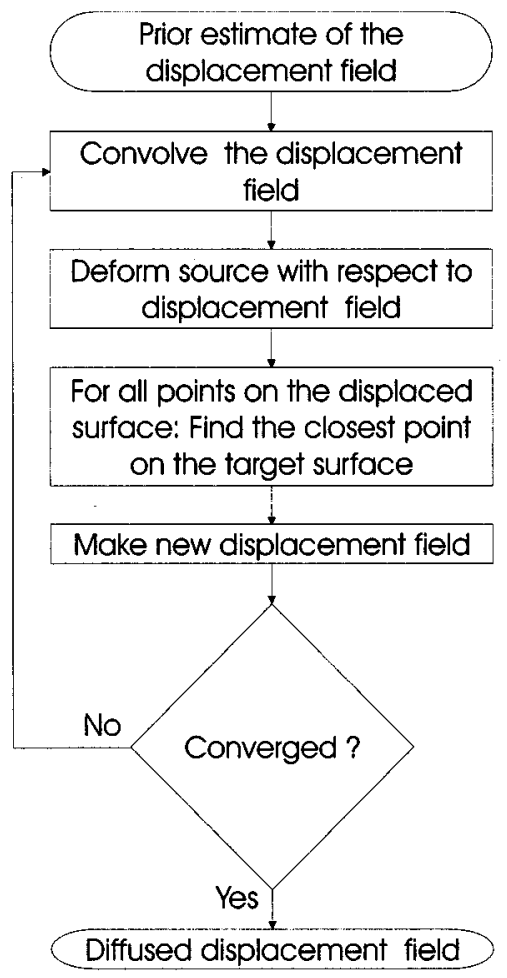

Fig. 5. Flow diagram for the diffusion algorithm. See Section V for details.
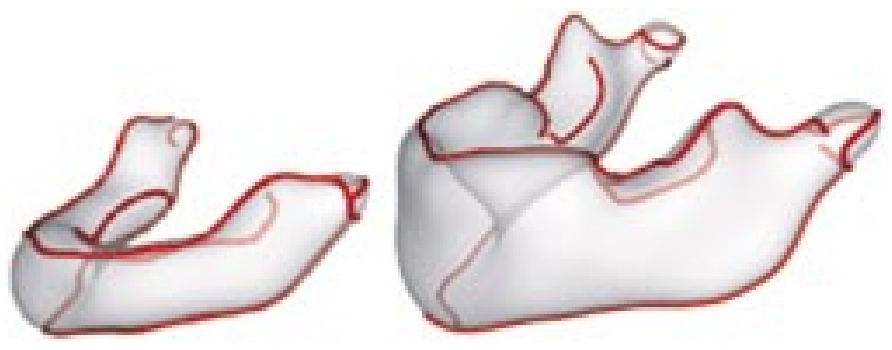

Fig. 6. Iso-surface and crest lines for a 3-mo-old and a 56-mo-old mandible: patient 1 , scan 1 , and patient 2 , scan 5 . The mandibles are Gaussian smoothed $(\sigma=3 \mathrm{~mm}$ ) in order to capture the higher scale features. The dimensions of the left and right mandibles are $(H \times W \times L) 18 \times 57 \times 53 \mathrm{~mm}$ and $31 \times 79 \times$ $79 \mathrm{~mm}$. Surfaces are translucent.

transformed to points in two Procrustes shape spaces (one for each gender). Relative warps are then used to search for trends in ontogenetic shape change. It is interesting that the result of the analysis is almost identical to the present work even though the 32 landmarks are placed mostly off the mandible, and the subjects are normal children.

We are not aware of any other growth studies in which longitudinal 3-D acquisitions from humans have been used for growth modeling except in [26]-[28], where the growth was modeled for the subject who is patient 6 here. Subsol [26] modeled the craniofacial growth using a linear model between a set of the controlling points. Bro-Nielsen et al. [27] used a nonrigid registration method to model the growth of the mandible. The method was a surface interpolation that did not preserve the mandibular shape; e.g., the condyles disappeared for intermediate interpolated time instances. Andresen et al. [28] used the same object registration technique as in Section IV
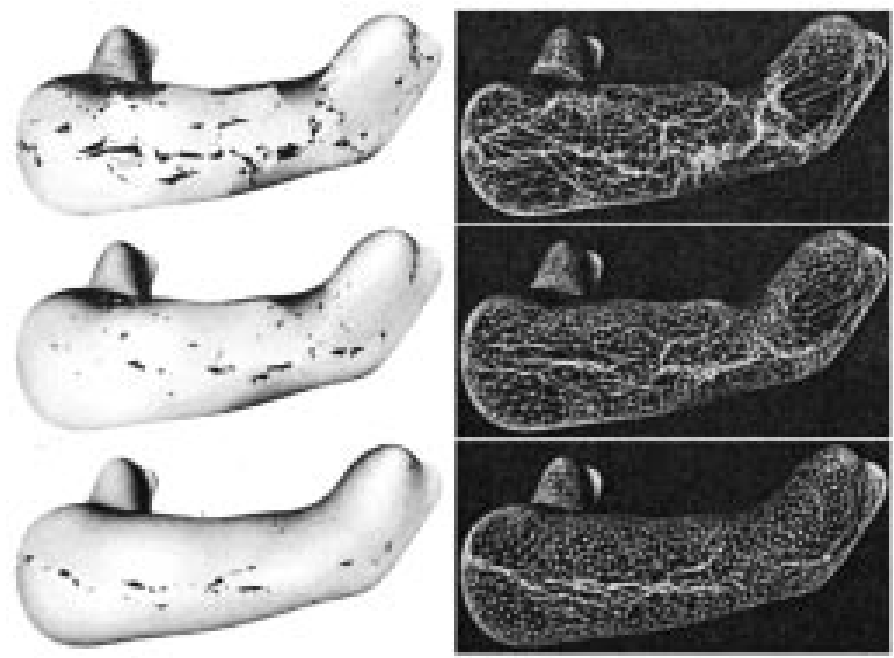

Fig. 7. Result of running the diffusion algorithm $\left(\sigma_{D}=2 \mathrm{~mm}\right)$ on the displacement field. The surface represents the 56-mo-old mandible after deformation to the 3-mo-old mandible in Fig. 6. The surface and wire frame of the deformed surface are shown to the left and right, respectively. The initial displacement, one iteration with the diffusion algorithm, and the last iteration are shown from top to bottom, respectively. The folds are a result of the imperfect initial registration whereby the extremal-mesh registration was extended to the whole surface by Gaussian regularization [28]. The final result is almost perfect, but some folds still exist, due to the discretization of the surface and displacement field.
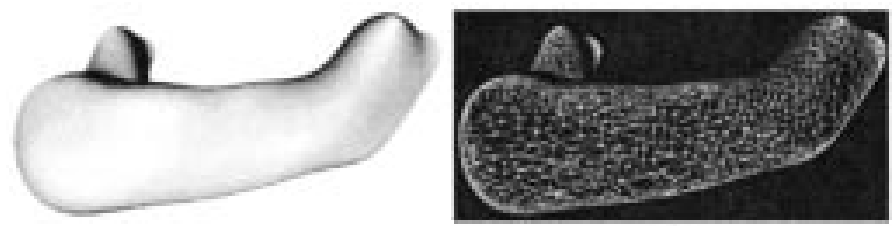

Fig. 8. Converged diffusion algorithm with a high value of $\sigma_{D}\left(\sigma_{D}=10\right.$ $\mathrm{mm}$ ). The surface and wire frame of the deformed surface are shown to the left and right, respectively. All folds remaining in the surface at the bottom of the previous figure are now gone.

TABLE I

CT SCANS USED IN THE PRESENT StUdy.

THE 56-Month SCAN For PATIENT 2 Is THE ReFENCE MANDIBLE FOR THE SEMILANDMARK ANALYSES

\begin{tabular}{c|c|c|c|c|c|c|c|c}
\hline \multirow{2}{*}{$\begin{array}{c}\text { Patient } \\
\text { number }\end{array}$} & \multirow{2}{*}{ Sex } & \multicolumn{6}{|c}{ Age in month for scan number } \\
\cline { 3 - 9 } & & 1 & 2 & 3 & 4 & 5 & 6 & 7 \\
\hline 1 & M & 3 & 16 & 21 & 23 & $\mathbf{3 4}$ & - & - \\
\hline 2 & M & 1 & 7 & 23 & 54 & 56 & 60 & 72 \\
\hline 3 & M & 1 & 5 & 17 & 32 & 36 & - & - \\
\hline 4 & F & 3 & 27 & 46 & 62 & 131 & 132 & 144 \\
\hline 5 & M & 3 & 4 & 21 & 72 & - & - & - \\
\hline 6 & F & 9 & 21 & 84 & - & - & - & \\
\hline
\end{tabular}

to register the mandibles. A second-order polynomial was used to interpolate the longitudinal displacement. Unfortunately, because the polynomial model there was not well-suited to mandibular growth modeling, the mandible was forecast to shrink in the course of growth!

\section{DATA MATERIAL}

The data consisted of CT scans of six subjects with Apert syndrome, four males and two females. Patient 6 is Danish, the 
TABLE II

PRINCIPAL COMPONENT ANALYSIS OF THE SEMILANDMARK DATA

\begin{tabular}{l|c||c|c||c|c}
\hline \multicolumn{3}{c||}{$\sigma=3, k=9087$} & \multicolumn{2}{c}{$\sigma=1, k=14851$} \\
\hline \multicolumn{2}{c|}{ no diffusion } & \multicolumn{2}{c|}{ diffusion } & \multicolumn{2}{c}{ diffusion } \\
\hline$\hat{\lambda}_{i} * 10^{5}$ & p.var & $\hat{\lambda}_{i} * 10^{5}$ & p.var & $\hat{\lambda}_{i} * 10^{5}$ & p.var \\
\hline 0.3134 & 45.19 & 0.3107 & 67.59 & 0.1897 & 62.18 \\
0.0496 & 7.15 & 0.0294 & 6.39 & 0.0222 & 7.27 \\
0.0415 & 5.99 & 0.0233 & 5.06 & 0.0164 & 5.33 \\
0.0373 & 5.39 & 0.0197 & 4.28 & 0.0151 & 4.95 \\
\hline
\end{tabular}
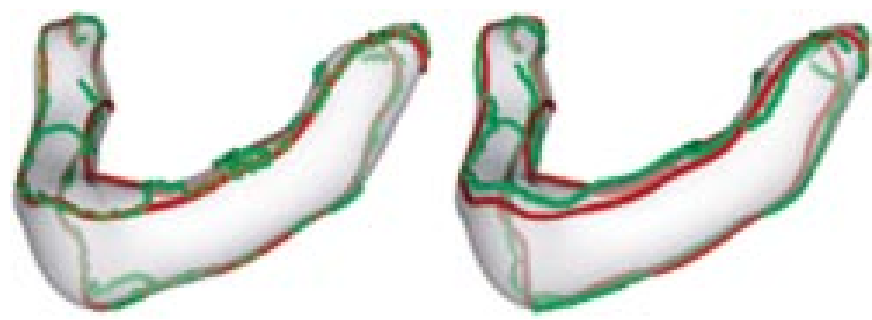

Fig. 9. Left and right images show the deformed (green) and the original (red) crest lines before and after applying the diffusion algorithm $\left(\sigma_{D}=2 \mathrm{~mm}\right)$. In the initial registration, crest lines are registered with crest lines. Where the topology does not change and away from umbilic points (for which the curvature is the same in all directions, e.g., at the condyles), (almost) no movement of the green crest lines is seen. Because erupting teeth change the topology on the upper surface (cf. Fig. 6), the green crest lines have moved in that region.

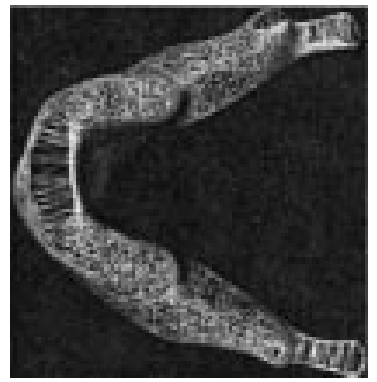

Fig. 10. The deformation vectors are moved too far away from the surface (the value of $\sigma_{D}$ is too high) resulting in a wrong projection back onto the surface.
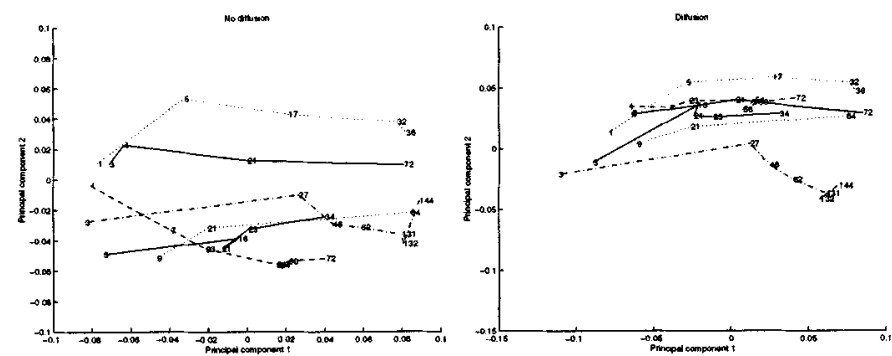

Fig. 11. Scatterplots of the first and second principal component for the six cases (left) before and (right) after the geometric-constrained diffusion is applied. For visual clarity, each patient's mean is restored. Numbers are ages in months. It is clear that the six growth trajectories span an angular range of nearly $90^{\circ}$ between the earliest growth segments of patient 2 (dashed line) and 3 (dotted line) in this projection; after diffusion (right), the alignment is far tighter.

others American. All scans were performed by Siemens scanners. The subjects were scanned for diagnostic, treatment planning, and follow-up purposes at the craniofacial clinics. The individual subjects were scanned between three and seven times, at ages between $1 \mathrm{mo}$ and $12 \mathrm{yr}$ (Table I).

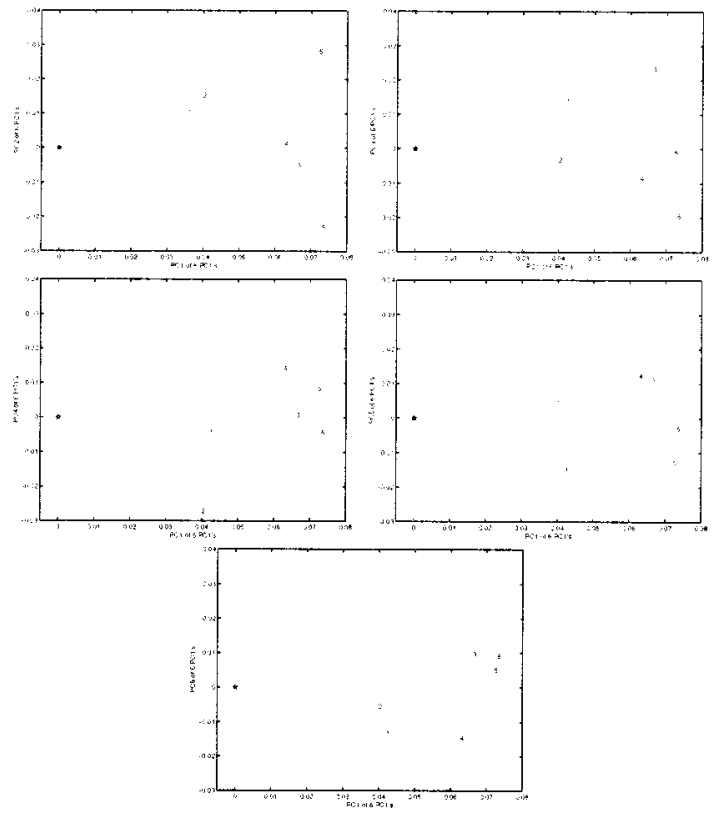

Fig. 12. The distribution of the six vectors (first principal component for each patient) in the six-dimensional (6-D) subspace they span.

TABLE III

CROSS-VALIDATION OF SHAPE PC1

\begin{tabular}{c|c|c}
\hline $\begin{array}{c}\text { Patient } \\
\text { excluded }\end{array}$ & $\begin{array}{c}\text { Percentage Variance explained } \\
\text { in model }\left(=100 \% * \lambda_{1} /\left(\sum_{i} \lambda_{i}\right)\right)\end{array}$ & $\alpha$ \\
\hline none & 62.2 & 0 \\
1 & 64.5 & 4.1 \\
2 & 65.8 & 5.8 \\
3 & 62.1 & 6.5 \\
4 & 62.3 & 8.3 \\
5 & 60.8 & 5.9 \\
6 & 61.1 & 3.9 \\
\hline
\end{tabular}

A previous two-dimensional roentgencephalometric study has shown that the mandible in Apert syndrome has relatively normal morphology except for some adaptive changes [29]. As these subjects are prepubscent and the sample is small, we have pooled the two sexes in the analysis to follow.

The mandibles were extracted from the CT scans by thresholding, with manual segmentation around the condyles. Holes inside the object were filled and the mandibles trilinearly resliced to $0.5-\mathrm{mm}$ cubic voxels (Fig. 1). The reference mandible from which we propagated all the semilandmark points in this study is the 56-mo scan for patient 2; the semilandmarks were chosen to be the vertices of the triangulated mandibular surface.

\section{REGiSTRATION: OBJECT CORRESPONDENCE}

In order to establish object correspondence, we search for features that match areas with equivalent morphology. Since the topology is not changed dramatically for the mandible when growing, features reflecting the "stable" geometry are used. The local shape of a surface is totally characterized by the principal curvatures $k_{1}, k_{2}\left(k_{1}>k_{2}\right)$ and their derivatives in the coordinate system defined by the principal directions $\left(t_{1}, t_{2}\right)$ [30]. 
We work with the extraction and matching of ridge lines [21], [28], [31].

Ridge lines come in four type types, corresponding to maximum or minimum Gaussian curvature $k_{1}$ and $k_{2}$. Here, we use the maximal $k_{1}$ type and the maximal $k_{2}$ type, called "crest lines" and " $k_{2}$-max lines," respectively. For three mandibles, these are shown in Fig. 2.

The overall framework follows the ideas originally proposed in [20] and already used in [28]. First, the crest lines and $k_{2}$-max lines for each dataset are extracted at a high scale, in order to get the more global features (Fig. 2). The crest lines are registered (Fig. 3) to provide a sparse initial deformation field. A dense field is then calculated by adaptive Gaussian filtering [32] (Section IV-A). The $k_{2}$-max lines are then deformed according to the initial dense deformation field and subsequently registered. From the two sets of matches (one from the crest lines, the other from the $k_{2}$-max lines) a combined deformation field is calculated. This sparse field is also interpolated to a dense field by adaptive Gaussian filtering. The resulting sparse deformation field is used as an initial guess in the point matching algorithm in Section V. Another iteration is done at a fine scale, but using the dense high-scale deformation field as initial displacement for the crest lines at fine scale.

This extremal mesh is manipulated in the course of registration first by a second-order moment registration to the target form (Section IV-B); then by two first-order polynomial deformations and two second-order polynomial deformations (Section IV-C); finally, by an unconstrained nonrigid deformation for all points on all lines (Section IV-D). Details of these steps are as follows.

\section{A. Adaptive Gaussian Filtering}

Gaussian filtering equals Gaussian smoothing for unevenly distributed points with a renormalization so that the total filter weight becomes unity. Adaptive Gaussian filtering [32] equals Gaussian filtering, but the scale parameter (the size of the Gaussian kernel) equals the square root of the distance to the nearest point.

\section{B. Second-Order Moment Registration}

The description in this section is adopted from [33]. The dispersion matrix for the mesh is given by

$$
\mathbf{D}=\frac{1}{L} \sum_{i=1}^{L}\left(\mathbf{q}_{i}-\overline{\mathbf{q}}\right)\left(\mathbf{q}_{i}-\overline{\mathbf{q}}\right)^{T}
$$

where

$\mathbf{q}_{i} \quad$ three-dimensional points in the mesh;

$\overline{\mathbf{q}} \quad$ center of mass for the points;

$L \quad$ number of points;

$(\cdot)^{T}$ transpose.

$\mathrm{D}$ is factorized by determining the eigensolution of the matrix.

$$
\mathrm{D}=\mathbf{P} \Lambda^{1 / 2}\left(\mathbf{P} \Lambda^{1 / 2}\right)^{T}
$$

where $\mathbf{P}$ is the orthonormal matrix of eigenvectors and $\Lambda$ is a diagonal matrix containing the eigenvectors.
The affine transformation $\left(\tilde{\mathbf{q}}_{T}=\mathbf{R} \mathbf{q}_{T}+\mathbf{t}\right.$, where $\mathbf{R}$ is the rotation matrix, $\mathbf{t}$ is the translation vector, and $\tilde{\mathbf{q}}_{T}$ is the transformed set) that gives set $T$ the same first and second-ordermatrix as set $S$ is determined by

$$
\begin{aligned}
\mathbf{R} & =\mathbf{P}_{S} \boldsymbol{\Lambda}_{S}^{1 / 2} \boldsymbol{\Lambda}_{T}^{-1 / 2} \mathbf{P}_{T}^{-1} \\
\mathbf{t} & =\overline{\mathbf{q}}_{S}-\mathbf{R} \overline{\mathbf{q}}_{T} .
\end{aligned}
$$

\section{C. vth-Order Polynomial Deformation, $v=1,2$}

Having the point correspondence between the two sets $S$ and $T$ (Section IV-E), the $v$ th-order polynomial deformation that optimally — in a least square sense-maps $T$ to $S$ is given by

$$
\langle\cdot\rangle_{\tilde{T}}=\sum_{i} \sum_{j} \sum_{k} a_{\langle\cdot\rangle_{i j k}} x^{i} y^{j} z^{k}, \quad i+j+k \leq v
$$

where $\tilde{T}$ is the mapped dataset and $\langle\cdot\rangle$ is one of the $x, y$, or $z$ coordinates. The parameters $a_{\langle\cdot\rangle_{i j k}}$ are determined from the correspondence between the two sets by least squares [34].

\section{Totally Nonrigid Deformation}

All the points are freely moved to their corresponding match in the other set (Section IV-E).

\section{E. Making the Point Correspondence}

The correspondence between meshes is determined by the ICP algorithm [22] modified as in [20] to take the line constraints into account.

\section{REGISTRATION: POINT CORRESPONDENCE}

From the sparse deformation field (the mapping between the two extremal meshes), derived from Section IV, a dense field must be calculated as we wish to register all the points on the whole surface, not only the extremal mesh. The points are the vertices of the triangulated mandibular surface. These points will be assumed to make up the semilandmarks after the geometry-constrained diffusion algorithm has been applied.

Adaptive Gaussian filtering can fold the mesh registration. To regularize the dense field, including simplifying (unfolding) it, we adopt the geometry-constrained diffusion algorithm originally published in [9] and [10].

The algorithm (Fig. 4) alternates Gaussian smoothing of the Cartesian components of displacement independently, which pulls them away from the surface, with projection back to the closest point on the surface. The detailed flow is as in Fig. 5; a typical example is set out in Figs. 6-8. Fig. 9 shows the improved registration achieved by the diffusion algorithm. In all cases, the "initial displacement field" is the dense deformation field (interpolated from the extremal mesh onto the whole surface) constructed in Section IV.

In the diffusion step, for each Cartesian component of the displacement field, a Gaussian weighted average is constructed. The standard deviation of the Gaussian $\sigma_{D}$ is the only parameter in the numerical scheme. In effect, it regulates the "time steps" between projections onto the surface. For simple surfaces, it may be large. For surfaces including regions of high 


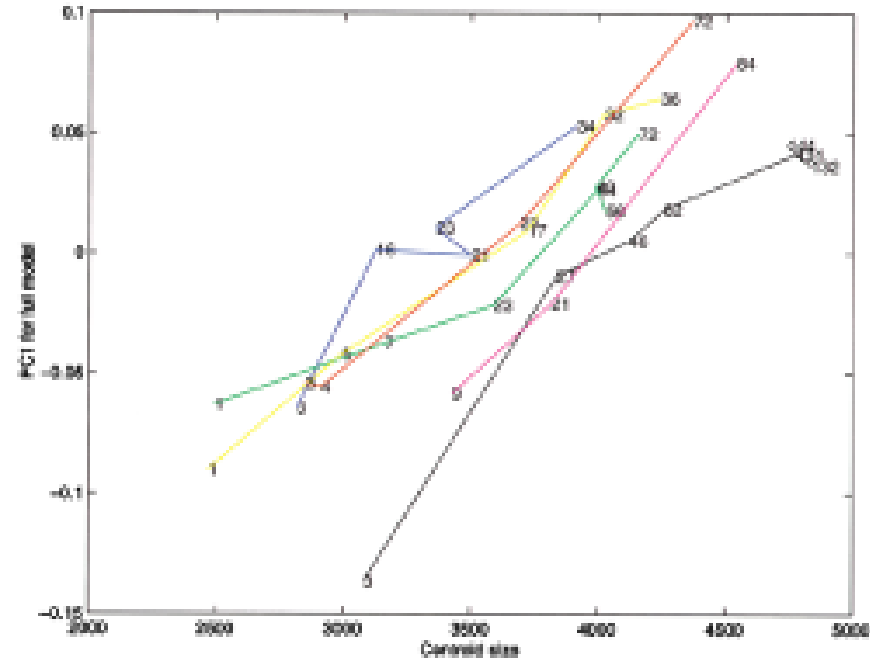

Fig. 13. CS against PC1, separately by patient. The numbers on the segmented lines are ages of observation. The correlation coefficient equals 0.83 (for all mandibles). The colors refer to the individual patients. The numbers on the lines are ages. See Section VII for further discussion.

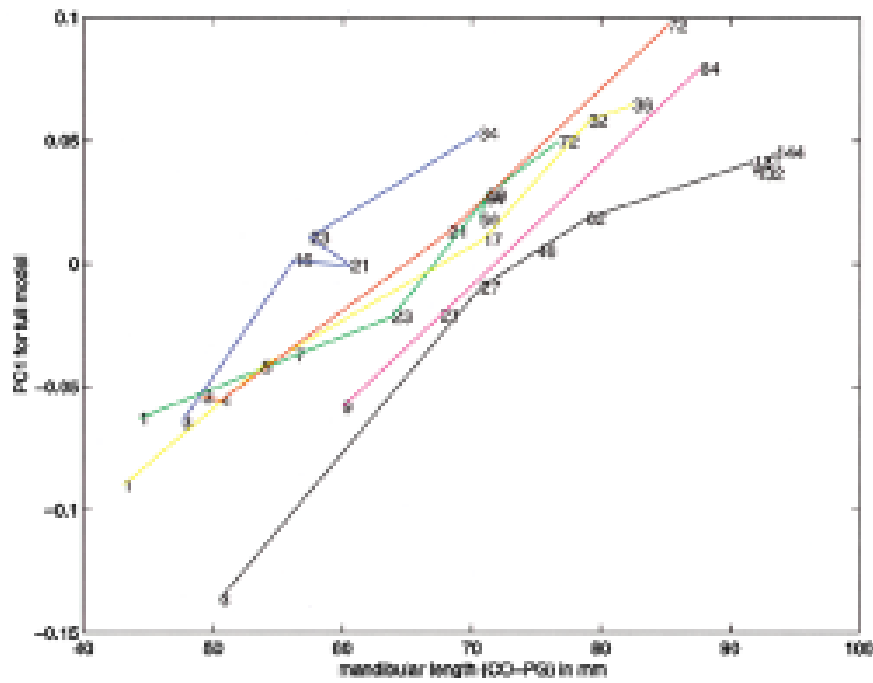

Fig. 14. The plot shows the length of the mandible calculated as the length between the midpoint of the most posterior superior point on the condylar heads and gnathion as done in [39]-Variable 79) versus the principal component for the full model. It is seen that there is a clear relation between shape and the length. The correlation coefficient equals 0.85 (for all mandibles).

curvature, $\sigma_{D}$ must be smaller in order not to tear the surface apart (see Figs. 7, 8, and 10). Theoretically, small values of $\sigma_{D}$ should yield the same result as larger values of $\sigma_{D}$ (when the surface is not torn apart), because of the additive nature of the Gaussian smoothing, but the discretization of the displacement field means that the algorithm could converge to a local optimum before a satisfactory result, i.e., a surface without folds, is obtained. A good approach is:

1) Set $\sigma_{D}$ to a small number, say one.

2) Run the algorithm.

3) If the result is satisfactory, then stop.

4) Increase $\sigma_{D}$, say $\sigma_{D}=\sigma_{D}+1$, go to 2).

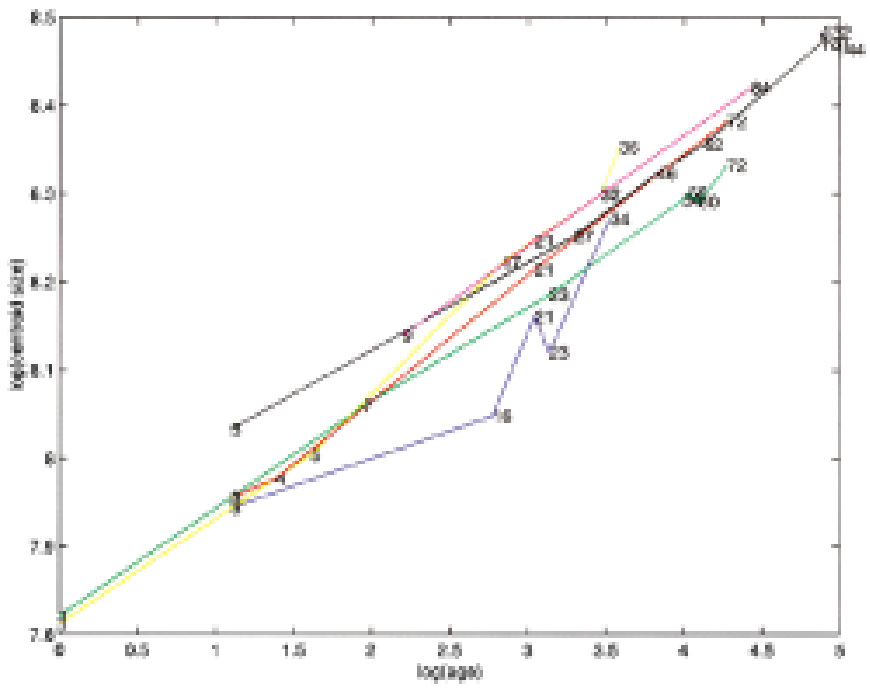

Fig. 15. The plot shows $\log ($ age $)$ versus $\log (\mathrm{CS})$ for the mandibles. The correlation coefficient is as high as 0.97 (for all mandibles) making it a 1-1 correspondence (almost) between the two variables.

The final surface registration is not sensitive to the value of $\sigma_{D}$, as long as the surface is not torn apart (Fig. 10). The influence of $\sigma_{D}$ is investigated further in [10].

For matching, as in [35], we use a $\mathrm{kD}$-tree [36] for finding the closest point on the target surface. As reference points on the triangulated target surface we use the center of mass $(\mathrm{CM})$ points of its triangles. The closest CM point is located (by the $\mathrm{kD}$-tree) and then the closest actual surface point is estimated as the intersection of the triangle's plane with the line through the reference point parallel to the target normal. If this intersection is outside the target triangle, its neighbors are searched. The proposed algorithm has the advantage that the points can move continuously on the target surface.

The diffusion is stopped when

$$
\sum_{p_{i}}\left\|D_{n}\left(p_{i}\right)-D_{n-1}\left(p_{i}\right)\right\|^{2}<\epsilon
$$

where

$p_{i} \quad$ points on the source surface;

$D_{n} \quad$ displacement in the $n$ th-iteration;

$\epsilon \quad$ user-chosen parameter.

Ten iterations are normally sufficient.

\section{Statistical Description: GEOMETRIC MORPHOMETRIC ANALYSIS}

In Section IV and Section V, our aim was to register the points on the 31 mandibles in such a way that we could assume that the points were semilandmarks. On the reference mandible the semilandmarks were chosen to be the vertices of the triangulated mandibular surface. The Gaussian-smoothed reference mandible has 14851 and 9087 vertices for the kernel sizes = 1 and $3 \mathrm{~mm}$, respectively. These meshes are deformed onto all other mandibles using the simplified dense deformation field set out in Section V. The semilandmarks that result are subjected 

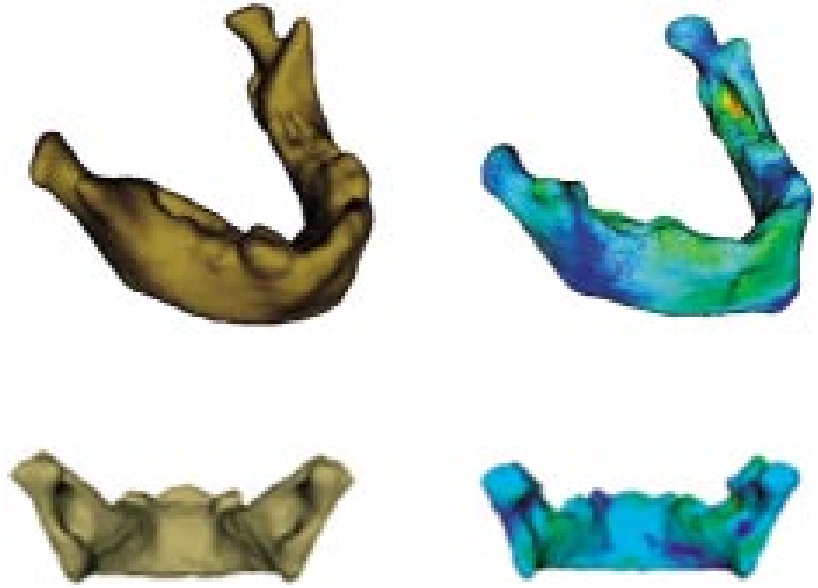

Fig. 16. Modeled last scan (scan \#5) based on the first scan (scan \#1) for patient 1. The large errors are located at the cavities as described in the text. The left images being the observed scan seen from two different viewpoints. The right images are the modeled mandible seen from the same two viewpoints. The surface is rainbow color coded from blue $(0 \mathrm{~mm})$ to red $(10 \mathrm{~mm})$ with the error (Fig. 23), calculated as the Euclidean distance between the same semilandmarks in the observed and modeled scan. The mean error is $2.4 \mathrm{~mm}$.
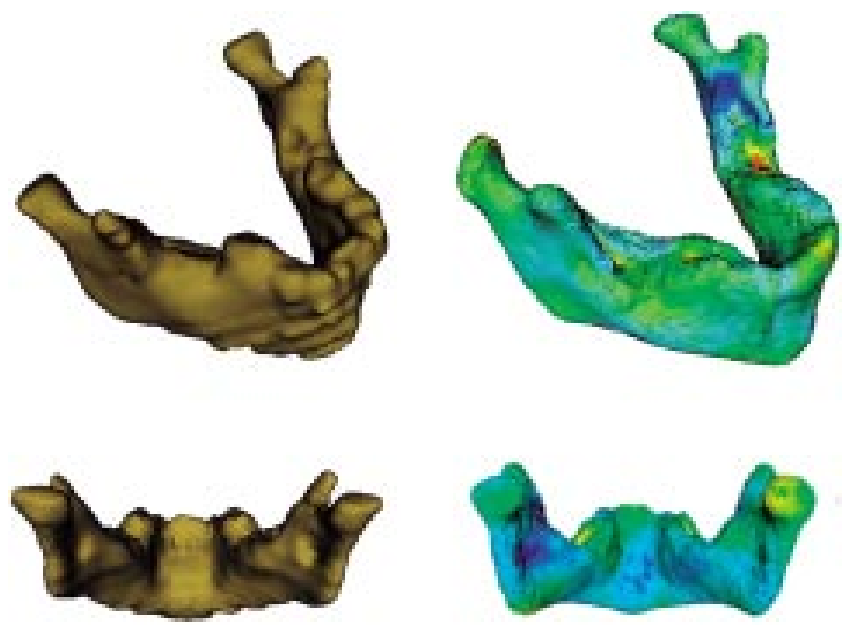

Fig. 17. Modeled last scan (scan \#7) based on the first scan (scan \#1) for patient 2. The large errors are located at the cavities introduced by the segmentation of the 1 -mo-old scan. The mean error is $3.5 \mathrm{~mm}$. The staircases on the observed scan \#7 is due to errors in the original volume.

to conventional multivariate biometric analyses that yield powerful predictors of unobserved (future) form.

At the outset, the configurations of 14851 semilandmarks, specimen by specimen, are aligned in a common coordinate framework by Procrustes analysis [7], [37], which is a leastsquares superposition in which position, orientation, and scale are jointly standardized. We used the usual iterative approach to produce an average shape $\overline{\mathbf{X}}$ to which the specimens are all aligned. Let $\mathbf{X}_{i}$ be the $k \times 3$ matrix of 3-D semilandmarks for the $i$ th specimen.

1) The center of mass (the centroid) for each shape is translated to the origin.

2) For each specimen, centroid size (CS) is computed, square root of the the summed squared distance of all semilandmarks from this centroid, and the form is rescaled so that this sum of squares becomes 1.0.

3) Make an initial guess at $\overline{\mathbf{X}}$, for instance, $\mathbf{X}_{\mathbf{1}}$.
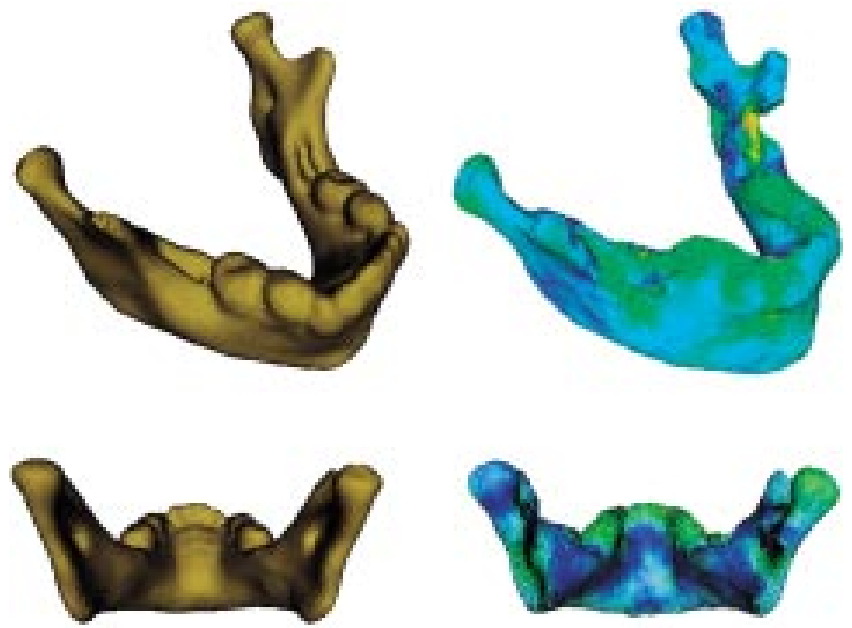

Fig. 18. Modeled last scan (scan \#5) based on the first scan (scan \#1) for patient 3. The mean error is $2.8 \mathrm{~mm}$. By looking at the first scan (Fig. 1) it is not surprising to find the large errors at the cavities. The notch in the side of the modeled mandible is related to the hole found at the same place on the first scan.

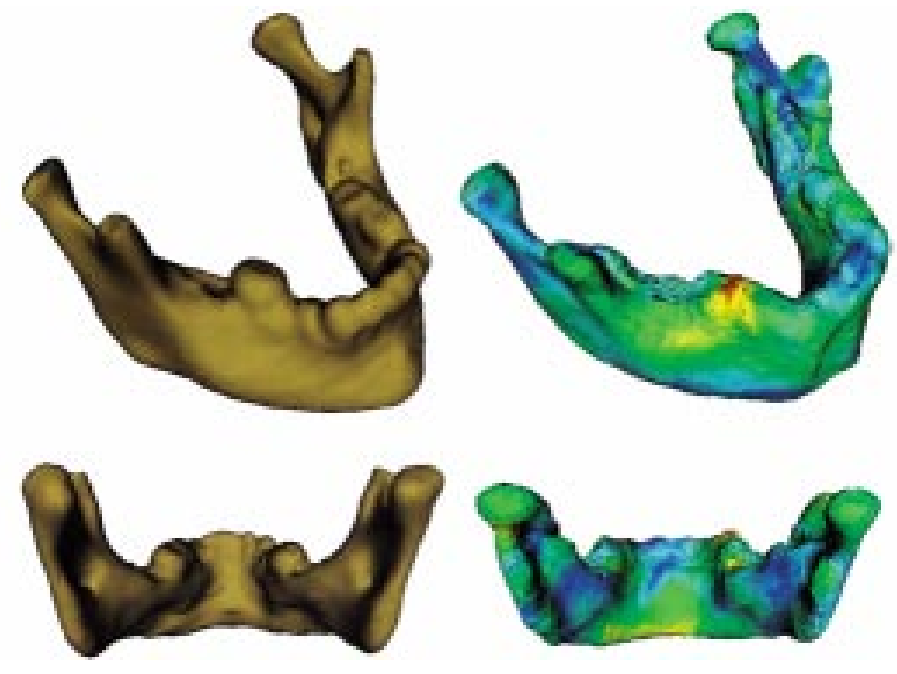

Fig. 19. Modeled last scan (scan \#7) based on the first scan (scan \#1) for patient 4 . The mean error is $3.7 \mathrm{~mm}$. The quality of the model is surprising. None of the scans in the model is as old as scan \#7 (12-yr old). The oldest scan in the model is 7-yr old (patient 6, scan \#3). This indicates the stability of the growth. The red area shows a missing tooth, which has been extracted. The prominent chin is not modeled very well. Using older scans solves the problem (Figs. 24 and 25).

4) Orient each shape to this tentative mean. To do so, let $U D V^{T}$ be the singular value decomposition of $\overline{\mathbf{X}}^{T} \mathbf{X}_{i}$. As long as all elements of $D$ are positive (which will be the case in all realistic applications), the rotation that superimposes $\mathbf{X}_{i}$ on $\overline{\mathbf{X}}$ is the $3 \times 3$ matrix $V U^{T}$.

5) Re-estimate the mean shape as the average of these rotated configurations.

6) Return to step 4) until convergence, which is invariably after two or three iterations.

Now that all the shapes are aligned, we calculate the patient mean shapes for each patient

$$
\overline{\mathbf{X}}_{i}=\frac{1}{n_{i}} \sum_{j=1}^{n_{i}} \mathbf{X}_{i, j}
$$



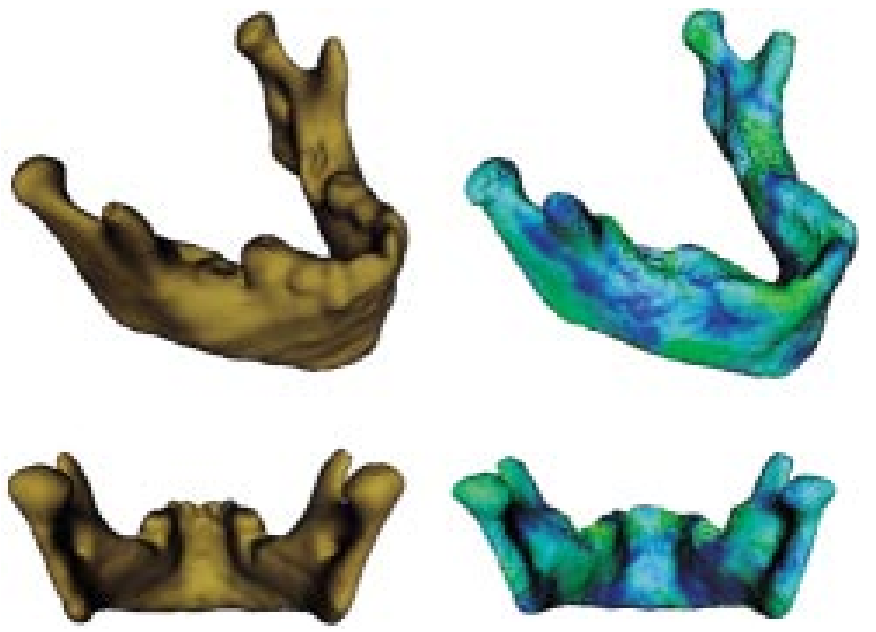

Fig. 20. Modeled last scan (scan \#4) based on the first scan (scan \#1) for patient 5 . The mean error is $2.6 \mathrm{~mm}$. An almost totally blue surface shows the high accuracy of the model.
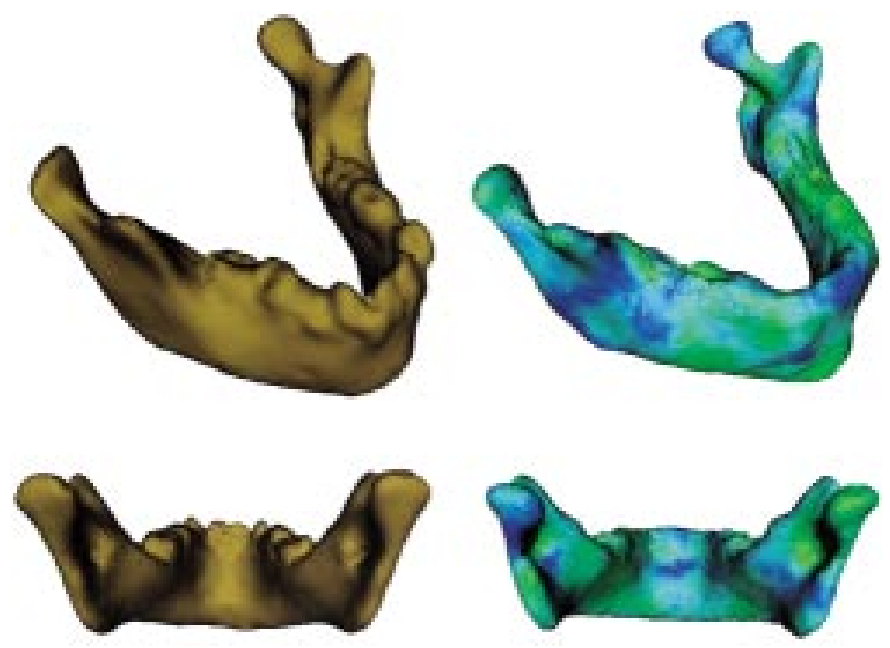

Fig. 21. Modeled last scan (scan \#3) based on the first scan (scan \#1) for patient 6 . The mean error is $2.8 \mathrm{~mm}$. The model is very accurate, only a slight twist of the condyles are seen.

where $n_{i}$ is the count of scans for the $i$ th patient. Write $\tilde{\mathbf{X}}_{p, i}$ for the patient-specific deviation from his/her own average

$$
\tilde{\mathbf{X}}_{p, i}=\mathbf{X}_{p, i}-\overline{\mathbf{X}}_{i}
$$

Rearrange each $\tilde{\mathbf{X}}_{p, i}$ into a column vector of length $3 k$, where $k$ is the count of semilandmarks (9087 or 14851 , depending on the computation), and concatenate all of these column vectors into a new data matrix $\tilde{\mathbf{X}}, 3 k \times 31$.

The covariance matrix of the patient-specific residual coordinates is $\mathbf{S}=\tilde{\mathbf{X}} \tilde{\mathbf{X}}^{T} / 30,3 k \times 3 k$. By the Eckart-Young's theorem [38], we can extract its eigenvectors very simply from the corresponding eigenvectors of $\mathbf{T}=\tilde{\mathbf{X}}^{T} \tilde{\mathbf{X}} / 30$, which is $31 \times$ 31.

Let $\tilde{\mathbf{e}}_{p}$ be the eigenvector of $\mathbf{T}$ with corresponding eigenvalue $\lambda_{p}$, sorted in descending order. It follows from [38] that the 31 vectors

$$
\mathbf{e}_{p}=\frac{1}{\sqrt{\lambda_{p}}} \tilde{\mathbf{X}} \tilde{\mathbf{e}}_{p}
$$
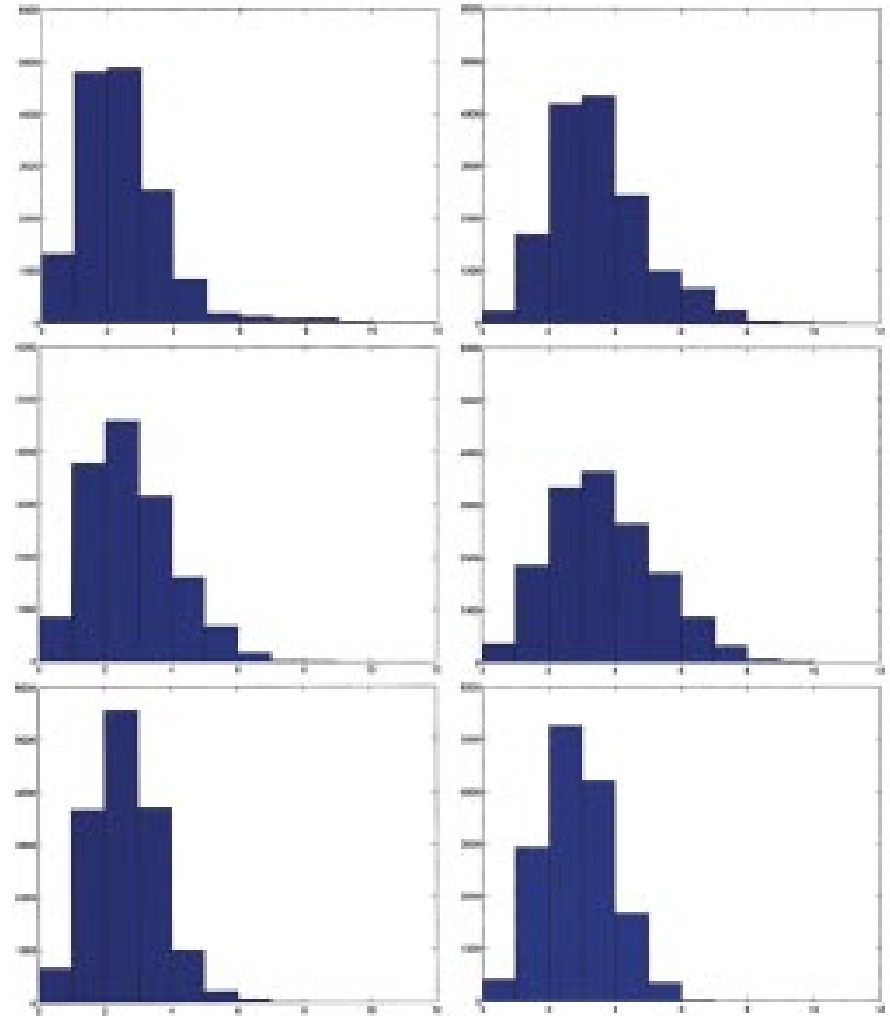

Fig. 22. Histogram of the prediction errors (Euclidean distance between the two same semilandmarks in the observed and modeled scan) for the six predicted mandibles shown in Figs. 16-21 (starting top left and ending bottom right). The larger error is mainly due to cavities coming from the segmentation of the very young scans (1-3 mo) as seen in Fig. 1.

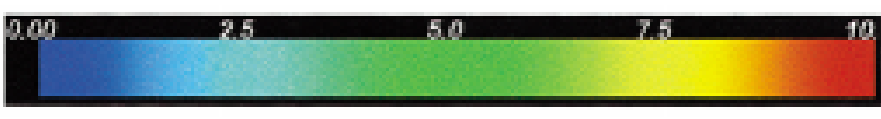

Fig. 23. Rainbow color-code from blue $(0 \mathrm{~mm})$ to red $(10 \mathrm{~mm})$ used to visualize the errors in the mandibular growth model.

are all eigenvectors of $\mathbf{S}$ with corresponding eigenvalues $\lambda_{p}$. The last $3 k-31$ eigenvectors are all zero.

\section{Shape EVAluation AND PREDiCTION}

Table II shows some results of these PCAs. There are two comparisons here: the effect of the surface-bounded diffusion algorithm (Section V), expressed in the difference of the two conditions for $\sigma=3$, and the effect of reducing the spatial scale $\sigma$ of the smoothing, observable in the relation of the diffusion with $\sigma=3$ to that with $\sigma=1$. For each set of 31 semilandmark configurations, the table shows the first four eigenvalues and the percentages of total Procrustes variation for which they account. The diffusion greatly strengthens the systematic aspects of the analysis; for instance, the shape variation along the first eigenvector increases by a factor of one and a half. Decreasing the size of the Gaussian smoothing radius $\sigma$, conversely, results in local differential changes that are less likely to be aligned across these 31 mandibles and, thus, a decrease of the explanatory power of these same eigenvectors.

Any summary of an ostensibly homogeneous sample of biological material, in this case, observed growth in six children 
with the same diagnosis of synostosis, is persuasive to the extent that that categorization "explains" the quantifications: the extent to which the extracted measurements are homogeneous over the class. From Fig. 11, it is clear that the six growth trajectories span an angular range of nearly $90^{\circ}$ between the earliest growth segments of patients 2 and 3 in this projection; after diffusion, the alignment is far tighter. As shown in Table II, there is far less variation around the common direction of these growth trajectories after diffusion than before (68\% vs. $45 \%)$.

From the right image in Fig. 11, it appears that the three to seven forms of each case lie reasonably close to a line in Procrustes space. We can, thus, summarize each by its own first principal component [16], and then examine the resulting six vectors to see how they may be ordinated.

Fig. 12 indicates the distribution of these six vectors in the 6-D subspace they span. Each principal component is normalized for its actual Procrustes variance explained, and the presentation here preserves that metric. We see a strong central tendency in these six growth vectors, closest to the observed trend for patient 3 or patient 4 . The variation of the tips of these six vectors seems to cover about the same diameter in the six different directions plotted here, without apparent correlation in the planes selected for plotting, and so it is reasonable to imagine it to be spherical, i.e., without a preferred directional structure within this space.

We demonstrate the power of this procedure by a series of growth predictions that, in all cases, predict the oldest form for each of the six patients by altering each of the earlier forms for that patient according to the regression on CS and the first principal component (PC1) given by the other five patients.

The model is built as described above except that one patient at a time, $m=1, \ldots, 6$, is excluded from the analysis. We will attend to the eigenvectors $e_{1, m}$ that arise as first eigenvectors of the data set excluding patient $m$ 's scans. As seen in Table III the growth models prove highly stable: fractions of Procrustes variance explained by the six eigenvectors $e_{1, m}$ range from $60.8 \%$ to $65.8 \%$ only, and the angle that these eigenvectors make with the pooled $e_{1}$ is limited to no more than $8^{\circ}$ (a cosine of 0.99 ). No patient controls the variability of the pooled analysis. Other computations, not shown here, confirm the observation, first set out in [16], that removing variation of patients' mean shape greatly enhances the reliability of the resulting PCA analysis of growth per se. Because patient $m$ 's scans are already aligned with the grand mean shape, a new shape may be modeled by

$$
\mathbf{x}_{\text {future }}=\mathbf{x}_{i}+t \cdot \mathbf{e}_{1, m}, \quad t \in \mathcal{R}
$$

where $t$ is a growth index parameter and $\mathbf{x}_{i}$ is one of patient $m$ 's earlier forms. If the new form is already available, the value of $t$ can be found by projection of the form onto the line of slope $\mathbf{e}_{1, m}$ through the earlier form. In a context of prediction instead, we can estimate an appropriate value of $t$ by regression using age or its transforms, CS, or mandibular length. These lead to equivalently accurate predictions (Figs. 13-15); we will use the one calibrated by CS, as that is the commonest in other applications of these Procrustes coordinates. Fig. 13 shows the clear relationship between $\mathrm{CS}$ and $\mathrm{PC} 1$ for the complete data
TABLE IV

Prediction Errors of Mandibular Shape. For Each Patient, the OldEst MANDIBUlar SHAPE Is PREDICTED FOR ITS CENTROID Size BY EACH EARLIER Form USING SHAPE PRINCIPAL COMPONENTS AND REGRESSIONS BAsed on the Other Five Patients of THE DATA SET

\begin{tabular}{c|c|c|c|c}
\hline \multicolumn{2}{c|}{ Patient } & \multicolumn{3}{c}{ Predicted shapes } \\
\hline number & scan & mean & std & rms \\
\hline 1 & 1 & 2.4196 & 1.2681 & 2.7318 \\
& 2 & 1.8005 & 0.7700 & 1.9582 \\
& 3 & 1.7475 & 0.7186 & 1.8895 \\
& 4 & 1.3569 & 0.5837 & 1.4771 \\
\hline 2 & 1 & 3.4783 & 1.4310 & 3.7612 \\
& 2 & 2.3849 & 1.1488 & 2.6472 \\
& 3 & 2.2891 & 1.2738 & 2.6196 \\
& 4 & 1.5070 & 0.7303 & 1.6746 \\
& 5 & 1.6217 & 0.7708 & 1.7956 \\
& 6 & 1.3683 & 0.6278 & 1.5054 \\
\hline 3 & 1 & 2.7568 & 1.2746 & 3.0372 \\
& 2 & 2.4617 & 1.1608 & 2.7217 \\
& 3 & 2.0740 & 0.9360 & 2.2754 \\
& 4 & 0.9720 & 0.4141 & 1.0565 \\
\hline 4 & 1 & 3.6691 & 1.5971 & 4.0016 \\
& 2 & 3.1307 & 1.5646 & 3.4999 \\
& 3 & 2.7982 & 1.4229 & 3.1392 \\
& 4 & 2.8315 & 1.3991 & 3.1583 \\
& 5 & 2.0300 & 0.9833 & 2.2556 \\
& 6 & 2.0289 & 0.9239 & 2.2294 \\
\hline 5 & 1 & 2.6153 & 1.0301 & 2.8109 \\
& 2 & 3.0812 & 1.2174 & 3.3130 \\
& 3 & 2.1441 & 1.0378 & 2.3821 \\
\hline 6 & 1 & 2.8292 & 1.0440 & 3.0157 \\
& 2 & 2.8013 & 1.2309 & 3.0598 \\
\hline & & & & \\
& & & & \\
& &
\end{tabular}

set of all 31 mandibles. To evaluate this prediction, of course we need to use the regression coefficient $\alpha$ based on the data set omitting the $m$ th case, the one we are predicting. The final model is, therefore

$$
\mathbf{x}_{\text {future }}=\mathbf{x}_{i}+\left(\mathrm{CS}_{\text {future }}-\mathrm{CS}_{i}\right) \cdot \alpha \cdot \mathbf{e}_{1, m} \text {. }
$$

From (11), it is seen that only one scan of the patient and the future CS or age (Fig. 15) is needed to make the prediction.

Table IV shows mean, standard deviation, and root-meansquared errors of surface prediction when each patient's last scan is predicted by each of his or her earlier scans at the appropriate CS, using predictions of size-related shape change based on the other five patients. In general, accuracy increases with decrease of age interval, but the prediction errors from earliest to latest scan seem gratifyingly homogeneous, in keeping with the evident parallelism of individual growth curves in Fig. 11.

Some of the predicted mandibles are shown in Figs. 16-21. The histograms of the errors for the figures are shown in Fig. 22. The left images in Figs. 16-21 represent the last observed scan for each patient. The right images show the modeled mandibles. The rainbow color coding shows the errors (Fig. 23). Error is the unsigned Euclidean distance between matched semilandmarks when the modeled surface is rescaled to match the observed scan in CS.

When segmenting the scans at 1-3 mo of age, large cavities are erroneously introduced because of the very low X-ray absorption (Fig. 1). As seen from Fig. 18, these errors are not removed by the growth model, as expected. For that reason, the largest errors $(>7 \mathrm{~mm})$ are seen at the cavities. Also, removal of a tooth is obviously not modeled, as seen in Fig. 19. Besides these very specific errors, we do not see errors larger than approximately $5 \mathrm{~mm}$, except for patient 4, scan \#7 (Fig. 19). This 

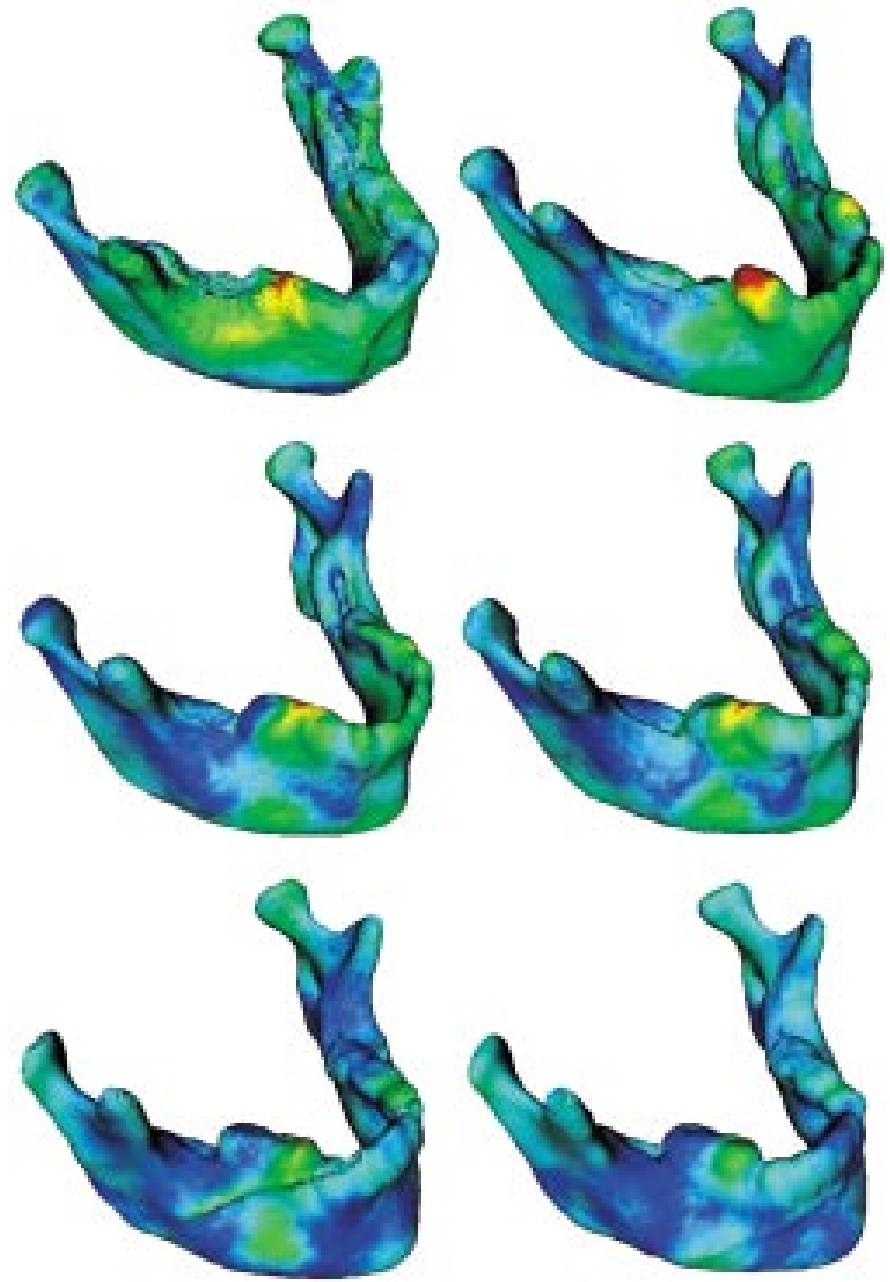

Fig. 24. Predicted shape of the most recent scan of patient 4 using each of her earlier scans, from top left to bottom right. The problem of cavities occurs only for the prediction from the earliest surface, and the accuracy of the prediction increases as the prediction interval becomes shorter. Notice, the model itself — the first eigenvector in (11)—stays the same.

patient, unlike the others, has a prominent chin, about which the prediction has no information.

The ability to model patient 4 , scan \#7 accurately is a bit surprising. None, of the other patients have been scanned at that age, the oldest scan (when patient 4 is excluded) being 7 -yr old (patient 6, scan \#3), but patient 4, scan \#7 is 12-yr old. This also indicates that the growth is modeled correct.

Figs. 24-25 are included to show how the errors decrease as successively older scans are used. Notice, how small the errors are when patient 4, scan \#6 (11-yr old) is used as the basis for the model. 12715 of the 14851 semilandmarks $(85.6 \%)$ have an error less than $3 \mathrm{~mm}$. Fig. 26 shows the histograms.

\section{CONCLUSION}

We have presented a linear 3-D growth model that relies on principal components of Procrustes shape coordinates. The model, which is formally independent of mandibular form per se [seen by subtracting $\mathrm{x}_{i}$ from (11)], is able to predict mandibular shape changes to an acceptable accuracy. The great increase in percent of Procrustes variance explained by the first eigenvector after geometry-constrained diffusion (Table II)
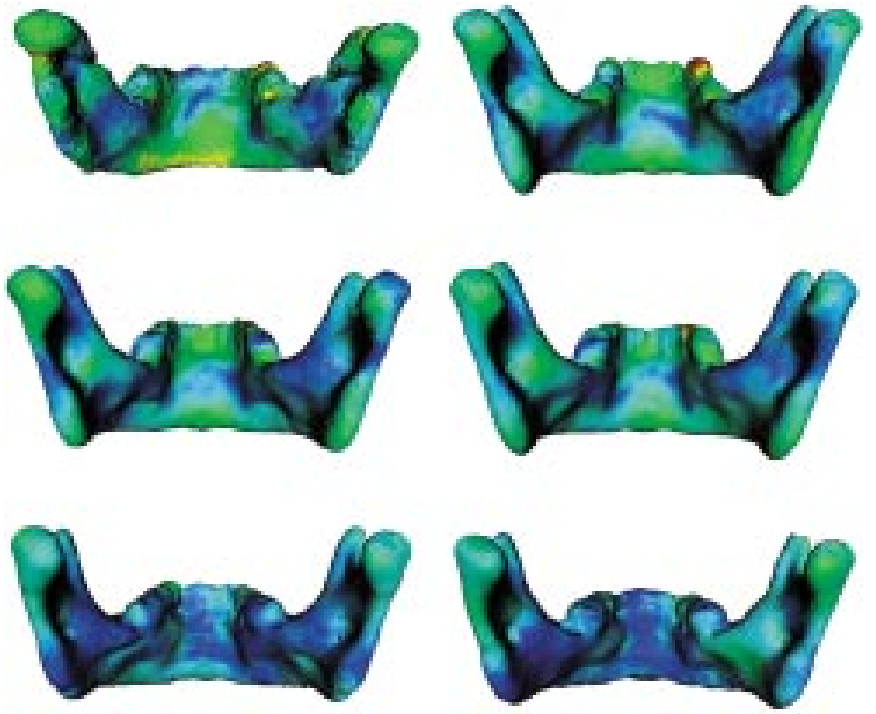

Fig. 25. The same six predicted mandibles, viewed from behind.
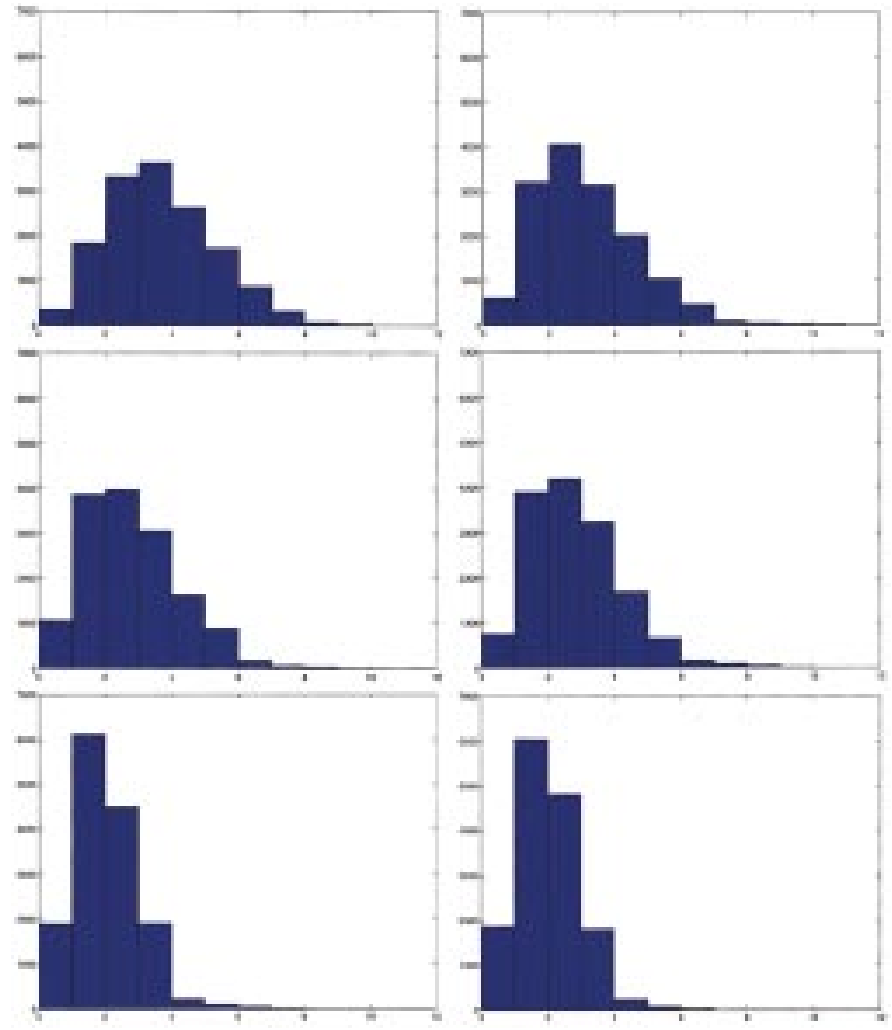

Fig. 26. Histograms for the errors of prediction in Figs. 24 and 25.

is surprising and deserves further investigation. Inclusion of zero-dimensional and one-dimensional constraints (landmark points and curves) would further improve this step in the processing.

Other studies, such as [40], show that the growth of the mandible is nonlinear, when using a "biological coordinate system." The present study does not reject that hypothesis, but indicates that the growth is linear if modeled in Procrustes space. This also complies very well with the result of [16]. A combination of the two "frames" might be very fruitful. Procrustes space could be used for the growth modeling and 
the "biological space" for the visualization. The ability to extrapolate the growth period by $5 \mathrm{yr}$ strengthens our hypothesis about linear growth.

At present, we can only obtain the closely spaces CT scans that allow this kind of analysis from clinical cases with various types of craniofacial growth disturbances. As mentioned above, in Apert syndrome, the mandible is not affected by the primary anomaly [29]. Other craniofacial syndromes also show fairly normal mandibular development e.g., Crouzon syndrome and achondroplasia. It will be interesting to ascertain which of these groups are characterized by these same mean growth trajectories. If the predictive accuracy we have demonstrated here extends to other syndromes lacking a primary mandibular dysmorphology, one might then speculate that it characterizes the normal growth process as well.

\section{ACKNOWLEDGMENT}

The authors would like to thank T. Darvann, 3D-Lab., Denmark, for bringing the CT data to Denmark. The authors are also grateful for the discussions with M. Nielsen, IT-University, Denmark. They would also like to thank the reviewers for their comments. The Visualization Toolkit (http://www.kitware.com) was used for the 3-D visualizations. Rendering was done by the Blue Moon Rendering Tools program rendrib (http://www.bmrt.org).

\section{REFERENCES}

[1] F. L. Bookstein, "Landmarks methods for forms without landmarks: Morphometrics of group differences in outline shape," Med. Image Anal., vol. 1, no. 3, pp. 225-243, 1997.

[2] H. Lester and S. R. Arridge, "A survey of hierarchical nonlinear medical image registration,” Pattern Recogn., vol. 32, pp. 129-149, 1999.

[3] J. B. A. Maintz and M. A. Viergever, "A survey of medical image registration,” Med. Image Anal., vol. 2, no. 1, pp. 1-36, 1998.

[4] A. K. Jain, Y. Zhong, and M.-P. Dubuisson-Jolly, "Deformable template models: A review," Signal Processing, vol. 71, pp. 109-129, 1998.

[5] T. McInerney and D. Terzopoulos, "Deformable models in medical image analysis: A survey," Med. Image Anal., vol. 1, no. 2, pp. 91-108, 1996.

[6] Advances in Morphometrics, ser. NATO Asi Series. Series A, vol. 284, 1996. Life Sciences.

[7] F. L. Bookstein, "Shape and the information in medical images: A decade of the morphometric synthesis," Comput. Vision Image Understanding, vol. 66, no. 2, pp. 97-118, 1997.

[8] J. Feldmar and N. Ayache, "Rigid, affine and locally affine registration of free-form surfaces," Int. J. Comput. Vision, vol. 18, no. 2, pp. 99-119, 1996.

[9] P. R. Andresen and M. Nielsen, "Non-rigid registration by geometryconstrained diffusion," Proc. Medical Image Computing and ComputerAssisted Intervention-MICCAI'99, pp. 533-543, 1999. vol. 1679 of Lecture Notes Comput. Sci.

[10] _ "Non-rigid registration by geometry-constrained diffusion," Med. Image Anal., 2000. Accepted for publication, to be published.

[11] F. L. Bookstein, Morphometric Tools for Landmark Data: Geometry and Biology. Cambridge, U.K.: Cambridge Univ. Press, 1991.

[12] M. Fleute and S. Lavallée, "Building a complete surface model from sparse data using statistical shape models: Application to computer assisted knee surgery," in Proc. Medical Image Computing and ComputerAssisted Intervention-MICCAI'98, 1998, pp. 879-887.

[13] R. Szeliski and S. Lavallée, "Matching 3-D anatomical surfaces with nonrigid deformations using octree-splines," Int. J. Comput. Vision, vol. 18, no. 2, pp. 171-186, 1996.
[14] K. Rohr, "Image registration based on thin-plate splines and local estimation of anisotropic landmark uncertainties," Medical Image Computing and Computer-Assisted Intervention-MICCAI'98, pp. 1174-1183, 1998. vol. 1496 of Lecture Notes Comput. Science.

[15] T. F. Cootes and C.J. Taylor, "A mixture model for representing shape variation," Image Vision Computing, vol. 17, no. 8, pp. 567-574, 1999.

[16] D. Dean, M. G. Hans, F. L. Bookstein, and K. Subramanyan, "Threedimensional bolton-brush growth study landmark data: Ontogeny and sexual dimorphism of the bolton standards cohort," Cleft Palate-Craniofacial J., vol. 37, no. 2, pp. 145-156, 2000.

[17] A. Kelemen, G. Székely, and G. Gerig, "Three-dimensional model-based segmentation of brain MRI," in Proc. Biomedical Image Analysis, 1998, pp. 4-13.

[18] L. H. Staib and J. S. Duncan, "Model-based deformable surface finding for medical images," IEEE Trans. Med. Imag., vol. 15, pp. 720-731, Oct. 1996.

[19] C. Brecbühler, G. Gerig, and O. Kübler, "Parametrization of closed surfaces for 3-D shape description," Comput. Vision Graphics Image Procession, vol. 61, pp. 154-170, 1995.

[20] G. Subsol, J.-P. Thirion, and N. Ayache, "A general scheme for automatically building 3-D morphometric anatomical atlases: Application to a skull atlas," Med. Image Anal., vol. 2, no. 1, pp. 37-60, 1998.

[21] J.-P. Thirion, "The extremal mesh and the understanding of 3-D surfaces," Int. J. Comput. Vision, vol. 19, no. 2, pp. 115-128, 1996.

[22] P. J. Besl and N. D. McKay, "A method for registration of 3-D shapes," IEEE Trans. Pattern Anal. Machine Intell., vol. 14, pp. 239-255, Feb. 1992.

[23] U. Grenander and M. I. Miller, "Computational Anatomy: An Emerging Discipline,", Tech. Rep., 1998. Center for Image Science (CIS).

[24] S. Joshi, "Large Deformation Diffeomorphisms and Gaussian Random Fields for Statistical Characterization of Brain Sub-Manifolds," Ph.D. dissertation, Washington Univ., St. Louis, MO, 1997.

[25] C. Lorenz and N. Krahnstöver, "Generation of point-based 3-D statistical shape models for anatomical objects," Comput. Vision Image Understanding, vol. 77, pp. 175-191, 2000.

[26] G. Subsol, "Construction automatique d'atlas anatomiques morphométriques à partir d'images médicales tridimensionnelles," Ph.D. dissertation, École Centrale, Paris, France, 1995.

[27] M. Bro-Nielsen, C. Gramkow, and S. Kreiborg, "Non-rigid image registration using bone growth model," in CVRMed-MRCAS'97: Springer, 1997, pp. 3-12. Lecture Notes Comput. Science.

[28] P. R. Andresen, M. Nielsen, and S. Kreiborg, "4D shape-preserving modeling of bone growth," Medical Image Computing and Computer-Assisted Intervention-MICCAI'98, pp. 710-719, 1998. vol. 1496 of Lecture Notes in Computer Science.

[29] S. Kreiborg, H. Aduss, and M. M. Cohen Jr., "Cephalometric study of the apert syndrome in adolescence and adulthood," J. Craniofacial Genetics Develop. Biol., vol. 19, pp. 1-11, 1999.

[30] M. P. do Carmo, Differential Geometry of Curves and Surfaces. Englewood Cliffs, NJ: Prentice-Hall, 1976.

[31] J. J. Koenderink, "The structure of images," Biological Cybern., vol. 50, pp. 363-370, 1984.

[32] M. Nielsen and P. R. Andresen, "Feature displacement interpolation," in Proc. IEEE 1998 Int. Conf. Image Processing (ICIP'98), 1998, pp. 208-212.

[33] R. Bajcsy and S. Kovacic, "Multiresolution elastic matching," Comput. Vision Graphics Image Processing, vol. 46, no. 1, pp. 1-21, 1989.

[34] W. H. Press, B. P. Flannery, S. A. Teulosky, and W. T. Vetterling, Numerical Recipes in C: Cambridge University Press, 1988.

[35] Z. Zhang, "Iterative point matching for registration of free-form curves and surfaces," Int. J. Comput. Vision, vol. 13, no. 2, pp. 147-176, 1994

[36] F. P. Preparata and M. I. Shamos, Computational Geometry. An Introduction. Corrected and Expanded Second Printing. Berlin, Germany Springer-Verlag, 1988.

[37] C. Goodall, "Procrustes methods in the statistical analysis of shape," $J$. Roy. Stat. Soc. B, vol. 53, no. 2, pp. 285-339, 1991.

[38] R. M. Johnson, "On the theorem stated by Eckart and Young," Psychometrika, vol. 28, pp. 259-263, 1963.

[39] M. L. Riolo, R. E. Moyers, J. A. McNamara Jr., and W. S. Hunter, An Atlas of Craniofacial Growth. Ann Arbor, MI: Center Human Growth Develop., Univ. Michigan, 1974.

[40] A. Björk and V. Skieller, "Normal and abnormal growth of the mandible. A synthesis of longitudinal cephalometric implant studies over a period of 25 years," Eur. J. Orthodontics, vol. 5, pp. 1-46, 1983. 